\title{
DYSL-X: Design of a tablet game for early risk detection of dyslexia in preschoolers
}

\author{
Lieven Van den Audenaeren', Véronique Celis², Vero Vanden Abeele1,3, Luc Geurts1,4, Jelle \\ Husson $^{1}$, Pol Ghesquière ${ }^{4}$, Jan Wouters ${ }^{5}$, Leen Loyez ${ }^{6}$, Ann Goeleven ${ }^{6}$ \\ 1 e-Media Lab, Group T - Leuven Engineering College (Association KU Leuven) \\ A.Vesaliusstraat 13, 3000 Leuven, Belgium \\ \{Lieven.vda@groept.be,vero.vanden.abeele,luc.geurts,jelle.husson\}@groept.be \\ 2 Parenting and Special Education, KU Leuven, L. Vanderkelenstraat 32, \\ box 3765, 3000 Leuven, Belgium \\ 3 CUO, IBBT/KU Leuven - Parkstraat 45, 3000 Leuven, Belgium \\ 4 ESAT, KU Leuven - Kasteelpark Arenberg 10, 3001 Heverlee, Belgium \\ 5 Experimental ORL, KU Leuven - Herestraat 49, box 721UO, 3000 Leuven, Belgium \\ 6 MUCLA, UZ Leuven, Kapucijnenvoer 33, 3000 Leuven, Belgium
}

\begin{abstract}
The goal of the DYSL-X project is to develop a tool to predict whether a preschooler (5 yrs) shows high risks for developing dyslexia. This tool is a tablet game that incorporates tests to take specific performance measures that allow for this prediction. The game will thus serve as an assessment tool to be used in school psychology services and clinical diagnostic and rehabilitation centers. In order to design the optimal tablet game for preschoolers, during the first phase of the projects several existing games for preschoolers were evaluated in order to derive design guidelines for games targeted at preschoolers. These design guidelines are presented in this paper and next, we show how these guidelines were used to develop the final game of the DYSL-X project.
\end{abstract}

\section{Introduction}

Dyslexia refers to specific problems to acquire reading and spelling skills despite adequate intelligence and instruction. It has a prevalence of $5-10 \%$ in the population [1,2]. The first signs of dyslexia are typically observed during the first year of formal reading education (age $7 \mathrm{yrs}$ ), but very often the diagnosis is only made at an older age. However, dyslexia is a developmental disorder that should be detected and treated as soon as possible. The younger the age of the child at the start of a therapy, the larger the long term effects. Early risk detection allows for taking preventive measures, which has been proven to be effective, even at preschooler age. 
Recent scientific studies provide us with a better understanding of predictive variables for dyslexia that can be measured, even in a preschool population [3-8]. The aim of DYSL-X is to integrate these predictors in a computer game, an aim to similar [9]. The underlying motivation of the DYSL-X project however is twofold. First, by using a game, preschoolers will display a higher motivation, a longer attention span, and as a result a more accurate measurement can be taken. Consequently, incorporating these measurements into a game will improve the quality of the tests and result in a more reliable and more valid prediction. Secondly, the DYSL-X game-based application will come with automated measurements and scoring. Therefore, no qualified personnel is needed to administer and score the tests. This allows for the deployment of DYSL-X at a wider scale, increasing the utilization potential. However, in order to develop the optimal assessment of the onset of dyslexia we need not only to understand how to measure the predictive variables, but equally how to design an optimal game experience for preschoolers, based on a thorough understanding of what constitutes fun for preschoolers. In this paper, we will describe the design process of the DYSL-x game based assessment tool, with the aim of achieving a high play motivation of preschoolers. We emphasize that in this paper we will not focus on the validation of the game, this will be the focus of forthcoming papers.

\section{Rationale of the project}

Existing dyslexia tests with preschoolers[4, 6-9] already aimed at gamification [10]. Researchers made the user interface more attractive and added animations in order to increase the motivation of the child $[5,11]$. However, the researchers conducting the gamified tests mentioned that it was still difficult to keep the child's attention at a high level throughout the test. It was suggested that a higher accuracy can be achieved by finding better ways to motivate the child. The challenge remains how to elicit a 'rich game play experience' and not just [12] coating while offering a reliable and valid test platform. It is important for game designers to address the true gratifications of this new gaming audience. As existing theoretical frameworks on meaningful gameplay for preschoolers are rare, dated and lack a comprehensive validation [13], we conducted a User Experience study (i.e. UX) Laddering $[14,15])$ to unveil the gameplay preferences of preschoolers. This study resulted in a list of meaningful and useful guidelines for future game designers, directed at this specific target group. In this workshop paper we present these design guidelines, and next, we demonstrate how we applied these to our own tablet game. 


\section{Method}

UX Laddering is an interview method that aims at unveiling the underlying motives for preferring one product over the other [14]. A comprehensive explanation of the method is besides the scope of this workshop paper, however, we refer the interested reader to the paper of Celis et al. [13] for more information. In short, 25 five-year olds were invited to play eight different games.

These digital games were selected in view of some predefined research questions that seemed interesting in the field of game design for preschoolers. For each element of interest, two games with contrasting features were selected. The first item related to the applied input device. Do preschoolers prefer to work on a tablet on the one hand or a laptop on the other hand? For this comparison, the child played the same game, i.e. Angry Birds, on both devices. Next, two different fantasy worlds were compared. In order to avoid gender biases, two comparison pairs were formulated. The first pair contained a dress-up game i.e. Dora DressUp, and a game of skill, i.e. K3 and the Ice Princess - Frog Game: Catiching Frogs. These games were in the first place marketed at girls and were both performed on a laptop. The next comparison pair contained two tablet games, that might appeal more to boys: a construction game, i.e. Toca Robot Lab, and a race game, i.e. Drawrace. Finally, we wanted to elucidate which narrative progression structure children prefer. Therefore two laptop games with a similar fantasy world were compared: a more linear adventure game, i.e. Putt-Putt, and a learning game with a hub world of different mini-games, i.e. Clifford. Consequently, four comparison pairs were obtained, which invited the child to make relative comparisons and judgments between two technologies.

The games were offered in pairs and preschoolers had to rank which game they preferred and explain why (Figure 1).

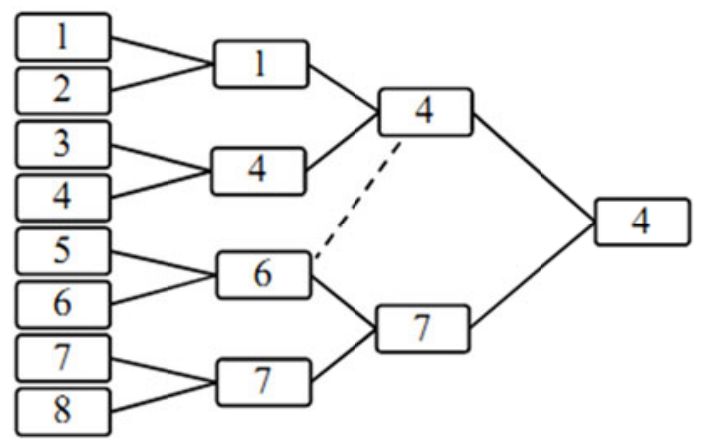

Fig. 1: Illustration of the preference ranking: eight different games were offered in pairs, preschoolers had to indicate which game they preferred. The preferred games were again paired, and preschoolers had to indicate their preference again. This 
procedure was repeated until the most favorite game was indicated, in total 7 pairs were offered.

\section{$4 \quad$ Results}

The following guidelines were derived from the study.

\subsection{Collecting Things $\gg$ Rewards/Winning $\gg$ Challenging}

Typically, for preschoolers the way to introduce challenge is by implementing goals and obstacles that require some form of problem solving (e.g. matching shapes, learning words, arithmetic's etc.). However, in our study this was not preferred. Instead, challenge was most favorably offered by 'collecting things'. Collecting things provided preschoolers with a sense of reward and victory and ultimately realized a challenging gameplay-experience.

\subsection{Touch Input vs. Mouse Input}

One of the contrasts we presented in this study related to the preferred input device. Consequently, preschoolers were offered games with touch input (via an iPad) as well as games with traditional mouse input. The test group preferred the touch input, in general, over the mouse input.

Furthermore, touch input was also linked to physical interaction. This shows that preschoolers like the intuitiveness of the physical interaction with their hands and fingers. Additionally, they simply seemed to like the physical movement.

Hence, implementing a mechanic of intuitive touch based control can contribute to achieving some kind of 'Sense-Pleasure'.

\subsection{Character Creation}

Character creation appeared to be the second most dominant element in our study (next to collecting things) and is found to be preferred by almost all children (22 out of 25).The children linked character creation to a relaxed, easy playing style, which implies that the preschoolers are not always looking for a 'challenging' gameplay. Other links with character creation were the opportunity for selfexpression, and an aesthetic sense. 


\section{$5 \quad$ Humorous, Destructive, Absurd and Visceral Effects}

Lastly, we would like to discuss the action-reaction effects that some games implement, i.e. the mouse is used to click on an object and in reaction something 'surprising or spectacular' happens. Effects like this are usually enjoyed by preschoolers, because they are perceived as humorous or satisfy curiosity. Destructive elements in effects are also perceived humorous, e.g. cutting out the enemy by destroying the castle in Angry Birds, as well as absurd elements (e.g. a dog with dresses). Below an overview of these findings is listed, translated into design guidelines.

Table 1: An overview of design guidelines

\begin{tabular}{ll}
\hline Aesthetics/gratifications & Mechanics/attributes \\
\hline Challenge & $\begin{array}{l}\text { Implement the collection of different items } \\
\text { through the game. This can equally serve as a } \\
\text { reward system and provides preschoolers with a } \\
\text { sense of victory. }\end{array}$ \\
Implement intuitive touch based controls that rely \\
on physical movement of the fingers, with natural \\
Arousal \\
Body and senses \\
mappings (i.e. analog to movement in real life). \\
Implement visceral effects and visual gags, \\
destructive elements (exploding items, breaking \\
walls,...). \\
Implement character creation, providing a \\
standard avatar, which can be outfitted with \\
different clothes and accessories. \\
This can be extended to the customization of all \\
kinds of objects, e.g. vehicles, houses etc. \\
expression \\
Implement humour via 'surprising or spectacular \\
effects' (e.g. deviating from the norm, preschoolers \\
already have a strong sense of how things should \\
be in reality.)
\end{tabular}

\subsection{Resulting design of the DYSL-X game}

The Diesel-X backbone story is about a robot dog with the name Diesel, who needs to get rid of the criminal gang of cats in the city. 


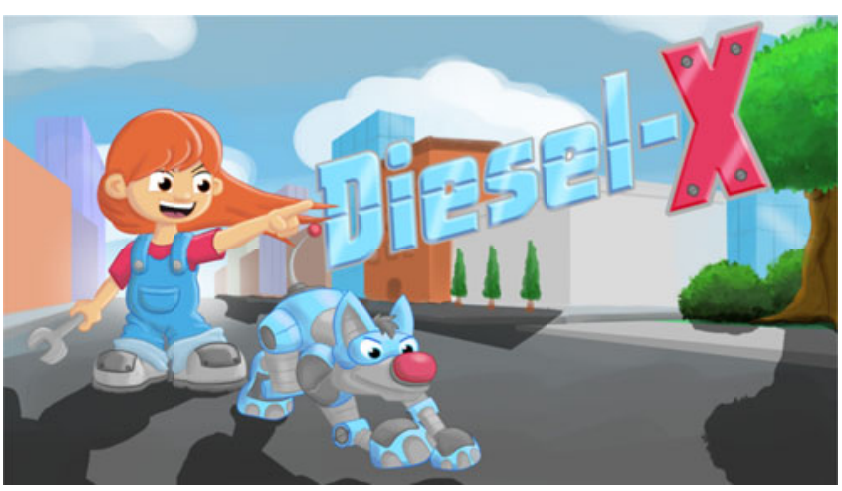

Fig. 2: Concept art for the Diesel-X story concept.

With the story of the game known, the actual game design started. The core of the design is a series of mini-games, each containing a performance test.

\subsection{The mini-games}

Diesel-X contains three mini-games at the moment: 1) a high-speed chase, 2) a lineup and 3) the search for lost objects. Each of these three mini-games incorporates one specific measurement related to the onset of dyslexia. Further details of these measurements are beyond the scope of this paper, however we refer the interested reader to [3-8] .

In the chase mini-game, Diesel is chasing cats that just robbed the bank. Each cat is holding a big bag, but only one contains the stolen goods. By stopping the cats and scanning the bags the player needs to catch the cat with the stolen goods.

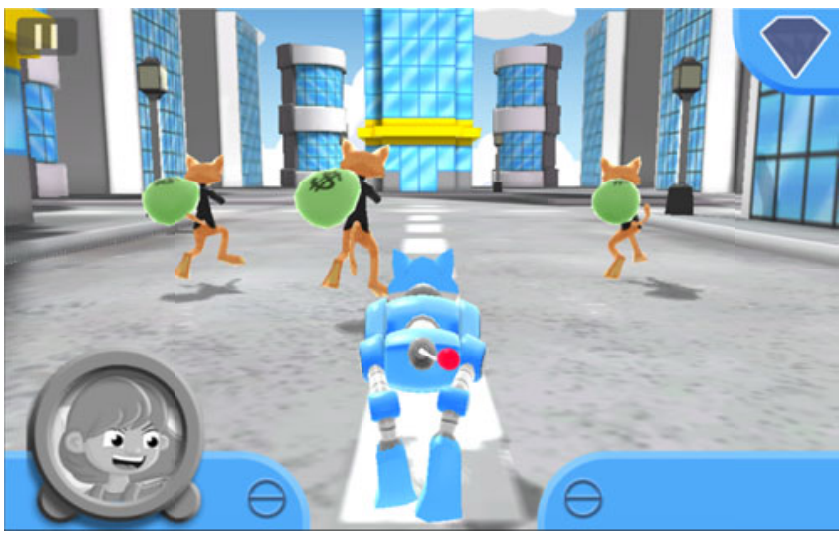

Fig. 3: Screen shot of the chase mini-game

The lineup mini-game is situated in the police office. Here a citizen comes to identify a criminal cat. Each cat holds a board with a letter on it and the citizen identifies the cat by calling the letter it is holding. The player then needs to select the right cat. 


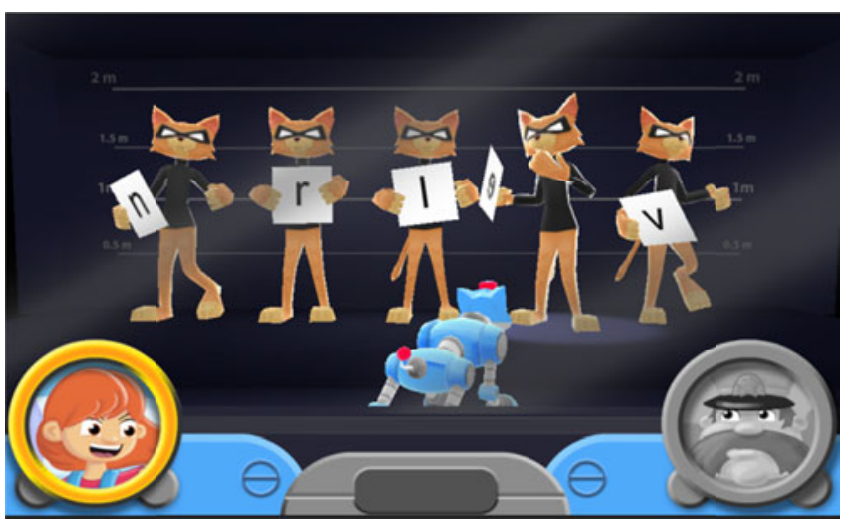

Fig. 4: Screen shot of the lineup mini-game.

The lost objects mini-game has Diesel searching for stashes of stolen goods. He needs to find specific items for Alex and she is playing a game with him. He needs to find an item that ends with the same letter as the item Alex is telling him about.

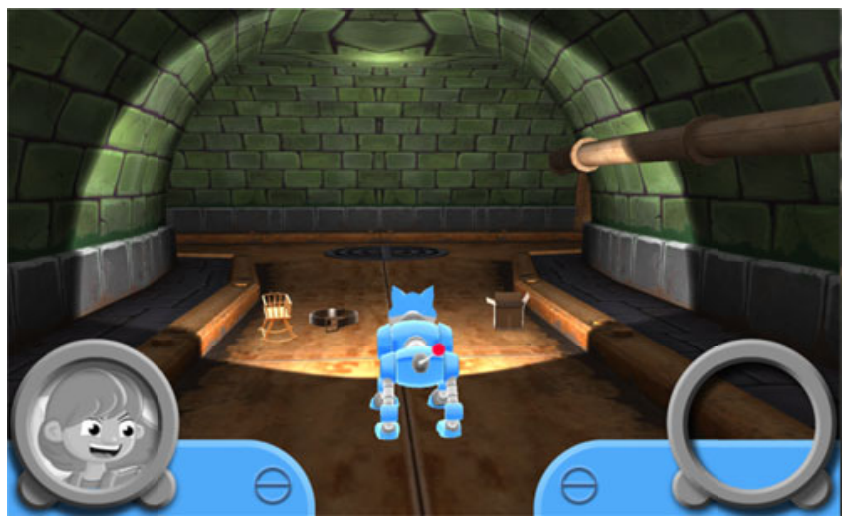

Fig. 5: Screen shot of the lost objects mini-game

The game design implements the design guidelines created by our previous study (Table 1).

\subsection{Collectible items}

The game implements collectible items in multiple ways.

Firstly, in the chase mini-game, the child can collect gold and even diamonds when chasing down the cat. Additionally, collecting things is also implemented through rewarding the player after each mini-game with an item that can be used in the character creation part. This way the player is rewarded for playing the performance tests by receiving more options for customizing Diesel.

Another implementation of collectible items is by rewarding the player with parts of a rocket after completing a series of mini-games, so that the cats can be sent to the moon at the end of the game. 


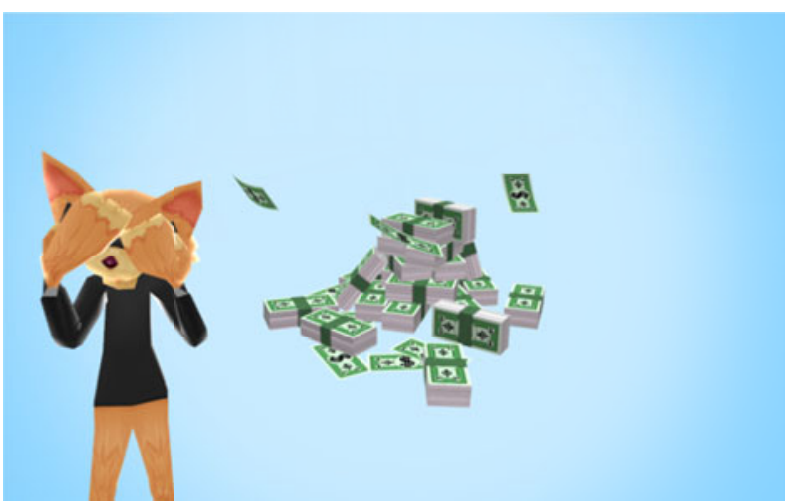

Fig. 6: Example of the money reward in the chase mini-game

\subsection{Touch-based intuitive control}

Based on the outcome of the study, we also decided that the target device of the game should be a tablet, which allows for touch-input. Although certain gestures can add value to the touch control, i.e. swiping or dragging, the complexity of the gestures in the performance tests is kept to a minimum, i.e. a simple tap. This is due to the fact that the results of the performance tests cannot be influenced by the experience the user has with the device.

\subsection{Character creation}

In between mini-games the player is able to customize Diesel in a character creation suite. Here it is possible to give Diesel a new paint job, customize him by adding a jet engine to make him fly, giving him a flash light for dark places etc... These customizations are earned by playing the mini-games.

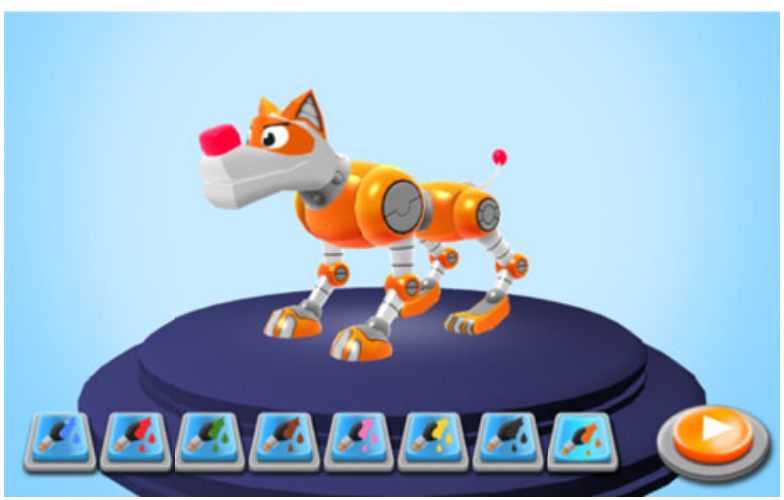

Fig. 7: Example of the character creation 


\subsection{Humor via deviating from the norm}

In the game, visual gags are used like big dust clouds and a screaming cat when Diesel is fighting with one of the criminal cats. Also when Diesel finds a bag with stolen goods, it can contain an item that does not belong there, thereby fooling the child player.

\section{Summary}

To summarize, the final goal of DYSL-X is the use of the game as an assessment tool for detection of children with high risks of developing dyslexia, in all school psychology services and clinical diagnostic and rehabilitation centers in Flanders. For the design of the game, we first conducted a study to create some design guidelines for our target audience (preschoolers) and used these to base our design on. During the workshop, we will present the intermediate results of the project.

\section{$7 \quad$ Acknowledgements}

We like to thank the teachers and preschoolers of Heilig Hart Heverlee, Scholengemeenschap De Kraal and Sint-Jan Berchmanscollege Diest who participated in our experiment with great enthusiasm.

\section{$8 \quad$ References}

1. Fawcett, A., Nicolson, R.: Dyslexia in Children: Multidisciplinary Perspectives. Harvester Wheatsheaf (1995).

2. Nicolson, R.I., Fawcett, A.: Dyslexia Early Screening Test. Pearson (2004).

3. Boets, B., Ghesquière, P., van Wieringen, A., Wouters, J.: Speech perception in preschoolers at family risk for dyslexia: Relations with low-level auditory processing and phonological ability. Brain Lang. 101, 19-30 (2007).

4. Boets, B., Wouters, J., van Wieringen, A., Desmedt, B., Ghesquière, $\mathbb{P} .:$ Modelling relations between sensory processing, speech perception, orthographic and phonological ability, and literacy achievement. Brain Lang. 106, 29-40 (2008).

5. Boets, B., Wouters, J., van Wieringen, A., Ghesquière, P.: Auditory temporal information processing in preschool children at family risk for dyslexia: Relations with phonological abilities and developing literacy skills. Brain Lang. 97, 64-79 (2006).

6. Boets, B., Wouters, J., van Wieringen, A., Ghesquière, P.: Coherent motion detection in preschool children at family risk for dyslexia. Vision Res. 46, 527-535 (2006). 
7. Boets, B., Wouters, J., van Wieringen, A., Ghesquière, P.: Auditory processing, speech perception and phonological ability in pre-school children at high-risk for dyslexia: A longitudinal study of the auditory temporal processing theory. Neuropsychologia. 45, 1608-1620 (2007).

8. Boets, B.: Early literacy development in children at risk for dyslexia. A longitudinal study of the general magnocellular theory., (2006).

9. Gaggi, O., Galiazzo, G., Palazzi, C., Facoetti, A., Franceschini, S.: A Serious Game for Predicting the Risk of Developmental Dyslexia in Pre-Readers Children. 2012 21st International Conference on Computer Communications and Networks (ICCCN). pp. 15 (2012).

10. Deterding, S., Dixon, D., Khaled, R., Nacke, L.: From game design elements to gamefulness: defining gamification. Proceedings of the 15th International Academic MindTrek Conference: Envisioning Future Media Environments. pp. 9-15 (2011).

11. Laneau, J., Boets, B., Moonen, M., van Wieringen, A., Wouters, J.: A flexible auditory research platform using acoustic or electric stimuli for adults and young children. J. Neurosci. Methods. 142, 131-136 (2005).

12. Malone, T.W., Lepper, M.R.: Making learning fun: A taxonomy of intrinsic motivations for learning. Aptit. Learn. Instr. 3, 223-253 (1987).

13. Celis, V., Husson, J., Abeele, V.V., Loyez, L., Van den Audenaeren, L., Ghesquière, P., Goeleven, A., Wouters, J., Geurts, L.: Translating preschoolers' game experiences into design guidelines via a laddering study. Proceedings of the 12th International Conference on Interaction Design and Children. pp. 147-156. ACM, New York, NY, USA (2013).

14. Zaman, B.: Introducing contextual laddering to evaluate the likeability of games with children. Cogn Technol Work. 10, 107-117 (2008).

15. Vanden Abeele, V., Zaman, B., De Grooff, D.: User eXperience Laddering with preschoolers: unveiling attributes and benefits of cuddly toy interfaces. Pers. Ubiquitous Comput. (2011).

16. Kevan, A., Pammer, K.: Predicting early reading skills from pre-reading measures of dorsal stream functioning. Neuropsychologia. 47, 3174-3181 (2009).

17. Kevan, A., Pammer, K.: Visual deficits in pre-readers at familial risk for dyslexia. Vision Res. 48, 2835-2839 (2008).

18. Franceschini, S., Gori, S., Ruffino, M., Pedrolli, K., Facoetti, A.: A causal link between visual spatial attention and reading acquisition. Curr. Biol. Cb. 22, 814-819 (2012).

Franceschini, S., Gori, S., Ruffino, M., Viola, S., Molteni, M., Facoetti, A.: Action Video Games Make Dyslexic Children Read Better. Curr. Biol. 23, 462-466 (2013). 